This is an electronic version of a paper Journal of Philosophical Logic 43: 979-997, 2014.

The following passage occurs on p.994 of the published version:

The invalidity of Antecedent Strengthening cannot be avoided by denying Substitution of Logical Equivalents. From A > C, Antecedent Strengthening gives $(A \wedge \sim C)>C$ which is false when $\sim C$ is compossible with $A$ (cf. Stalnaker [24]: 124). Now when $A$ is possible and irrelevant to whether or not $C$ obtains, we have a particularly vivid instance of this failure. In this case we have both the required premise, $A>C$, and also $A>\sim C$. But given that (i) $A>\sim C$ requires that $A$ and $\sim C$ be compossible, unless $A$ is impossible, and (ii) nothing in the example requires that $A$ be impossible, then there will be instances where $A$ and $\sim C$ are compossible, and so instances where $(A \wedge \sim C)>C$ is false. Footnote 18: This argument relies on the following truth about counterfactuals: $(A>C) \supset(\diamond A \supset \diamond C)$. Given Reflexivity and Agglomeration, this rules out the truth of $(A \wedge \sim C)>C$ when $A$ and $\sim C$ are compossible. Again given Reflexivity and Agglomeration, it also entails that $A$ and $\sim C$ are compossible given both $A>\sim C$ and $\diamond A$.

Obviously, something has gone wrong here since we only have $A>C$ and $A>\sim C$ when $A$ is impossible! What the passage should say is:

The invalidity of Antecedent Strengthening cannot be avoided by denying Substitution of Logical Equivalents. From A > C, Antecedent Strengthening gives $(A \wedge \sim C)>C$ which is false when $\sim C$ is compossible with $A$ (cf. Stalnaker [24]: 124). Now when $A$ is irrelevant to whether or not $C$ obtains, and $C$ is irrelevant to whether or not $A$ obtains, we have a particularly vivid instance of this failure. In this case we have both $A>C$ and $\sim C>A$. But given that (i) $\sim C>A$ requires that $\sim C$ and $A$ be compossible, unless $\sim C$ is impossible and (ii) nothing in the example requires that $(\sim C \wedge A)$ be impossible, then there will be instances where $A$ and $\sim C$ are compossible, and so instances where $(A \wedge \sim C)>C$ is false. Footnote18: This argument relies on the following truth about counterfactuals: $(A>C) \supset(\vee A \supset \diamond C)$. Given Reflexivity and Agglomeration, this rules out the truth of $(A \wedge \sim C)>C$ when $A$ and $\sim C$ are compossible. Again given Reflexivity and Agglomeration, it also entails that $A$ and $\sim C$ are compossible given both $\sim C$ $>\mathrm{A}$ and $\diamond \sim \mathrm{C}$.

This has been corrected in the version below. 


\section{Against Hypothetical Syllogism}

\section{Lee Walters}

The debate over Hypothetical Syllogism is locked in stalemate. Although putative natural language counterexamples to Hypothetical Syllogism abound, many philosophers defend Hypothetical Syllogism, arguing that the alleged counterexamples involve an illicit shift in context. The proper lesson to draw from the putative counterexamples, they argue, is that natural language conditionals are context-sensitive conditionals which obey Hypothetical Syllogism. In order to make progress on the issue, I consider and improve upon Morreau's proof of the invalidity of Hypothetical Syllogism. The improved proof relies upon the semantic claim that conditionals with antecedents irrelevant to the obtaining of an already true consequent are themselves true. Moreover, this semantic insight allows us to provide compelling counterexamples to Hypothetical Syllogism that are resistant to the usual contextualist response.

Keywords: conditionals; context-sensitivity

For which interpretations of the conditional, $\Rightarrow$, is

Hypothetical Syllogism: $((A \Rightarrow B) \wedge(B \Rightarrow C)) \supset(A \Rightarrow C)$

valid $?^{1}$ It is controversial which, if any, natural language conditionals validate Hypothetical Syllogism, but putative counterexamples have been suggested for both counterfactual and indicative conditionals. These counterexamples have not been universally accepted,

${ }^{1} \mathrm{~A}$ note on symbolism. ' $\Rightarrow$ ' is a variable ranging over various conditionals, so sentences containing it should be assessed relative to an interpretation, that is, for some particular conditional. I reserve ' $\supset$ ' for the material conditional, and '>' for counterfactual conditionals. 
however, and the current state of the debate is marked by one side providing a putative counterexample to Hypothetical Syllogism, and the other side invoking changes in context to explain it away. Consider the following case from Wright (1983):

1. If there had been snow in the valley yesterday, I would have gone skiing.

2. If an avalanche had taken place yesterday, there would have been snow in the valley yesterday.

If Hypothetical syllogism is valid, then (1) and (2) license

3. If an avalanche had taken place yesterday, I would have gone skiing.

But it seems as if (1) and (2) might well be true and yet (3) false.

Stalnaker (1968) takes examples like this to show that Hypothetical Syllogism is invalid for both counterfactuals and indicatives, and Lewis (1986) agrees in the case of counterfactuals. Wright demurs, noting that if I were to utter (1) and (2) "in the same breath" then you'd have "cause to wonder whether I had indicated my willingness to undertake a suicidal skiing trip" and would "be bound to wonder ... whether I had meant to hint that I had been depressed, or some such thing" (1983: 138). But if (1) to (3) represent a counterexample to Hypothetical Syllogism "then there ought in general to be nothing incongruous about a subject's assertion, 'in the same breath', of [(1) and (2)]; incongruity should arise only when the step is invalidly, made" (Wright 1984: 184). So for Wright, Hypothetical Syllogism is valid, since any context in which (1) and (2) are jointly assertable is also one in which (3) is assertable. The apparent invalidity of Hypothetical Syllogism is, for Wright, an illusion underwritten by changing contexts: in most cases, (1) is true in a first context, (2), in a second, but (3) true in neither. 
However, Wright claims that if (1) and (2) are true in a single context, then their conjunction does seem to indicate my willingness to ski even in the event of an avalanche.

Wright clearly has a point, and similar things can be said about the examples to which Stalnaker and Lewis appeal in their case against Hypothetical Syllogism. For example, Stalnaker and Lewis present versions of the following to undermine Hypothetical Syllogism:

4. If Hoover had been born in Russia, he would have been a communist.

5. If Hoover had been a communist, he would have been a traitor.

From (5) and (6), by Hypothetical Syllogism, we have

6. If Hoover had been born in Russia he would have been a traitor.

Lewis and Stalnaker take (4) to (6) to represent a counterexample to Hypothetical Syllogism, since whereas (4) and (5) seem true, (6) seems false. But Lowe disagrees, saying of a structurally similar example that:

it seems more plausible to suggest that what is wrong with [(4) to (6)] has nothing to do with the form of the inference employed, but rather something to do with the difficulty of finding an intelligible conversational context for the co-assertion of the premises. ... [And we have] a strong intuition that the fallacy involved in [the above inference] is some sort of fallacy of equivocation (Lowe: 1990: 81).

Again, there is something to Lowe's point, as conjoining (4) and (5) does seem odd. As von Fintel puts it: 
We are not at all tempted to admit both premises as true [simultaneously]. The natural way of reading the second premise is as taking into account a set of Communist scenarios including those introduced by the first premise. With respect to the active context, then, the second premise is naturally read as a expressing a proposition that is in fact false (von Fintel 2001: 132). ${ }^{2}$

The debate over the validity of Hypothetical Syllogism is, then, currently in stalemate, with Lowe, an advocate of Hypothetical Syllogism, recently writing that the "dispute will never be conclusively settled merely by piecemeal appeal to alleged 'counter-examples', but only, if at all, on the basis of broader, systemic considerations" (Lowe 2010: 289). I agree, in part, with Lowe, and in this paper I try to move the debate on by appeal to systematic considerations about conditionals, and also by providing new-style counterexamples to Hypothetical Syllogism which such considerations motivate.

In §1 I consider and reject an attempt of Lowe's to motivate Hypothetical Syllogism which rests on general considerations. I then present Morreau's reductio of Hypothetical Syllogism which employs four plausible principles of conditional logic. The weakness of Morreau's reductio is that it relies on a principle of counterfactual logic which is controversial and which the defender of Hypothetical Syllogism rejects (§2). With a view to improving upon Morreau's reductio, I outline a general semantic feature of counterfactuals (§3), namely that a conditional with a true consequent, and an antecedent irrelevant to the obtaining of the consequent is true. Then, in §4-5, I present two revised reductios of Hypothetical Syllogism for counterfactuals which exploit the semantic feature discussed in $\S 3$ instead of the controversial principle of conditional logic employed by Morreau. Next, I respond to an objection to the proofs of $\S 4-5$ inspired by Lowe (§6). In $\S 7$ I provide two improved proofs of

\footnotetext{
${ }^{2}$ I am here prescinding from the details of how shifts in contexts are affected on different contextualist views. I simply want to motivate the contextualist response to putative counterexamples to Hypothetical Syllogism so that I can sketch the current shape of the debate.
} 
the invalidity of Hypothetical Syllogism for counterfactuals each of which relies only upon the semantic claim of §3. To add credence to the abstract proofs appealed to so far, I provide new-style counterexamples to Hypothetical Syllogism for which there is no work for a contextualist response to do (§8). I then (§9) argue that the same considerations also show the invalidity of Antecedent Strengthening and Contraposition for counterfactuals. Finally, in $\S 10$, I consider whether the proofs can be extended to the case of indicative conditionals, concluding that, although not as decisive in this case, we have still improved upon Morreau's initial proof.

\section{Lowe's Reduction of Conditional Logic}

As well as arguing that putative counterexamples to Hypothetical Syllogism are to be rejected, Lowe provides one general argument to the effect that Hypothetical Syllogism is valid. Lowe $(1983,1995)$ argues that we can reduce the logic of conditionals to the logic of modality by defining the conditional as follows: $A \Rightarrow C=d f \square(A \supset C) \&(\diamond A \vee \square C)$ (where $A \Rightarrow$ C can be treated as either an indicative or as a counterfactual conditional). Lowe's account has the advantage that it is simple, reductive, and validates a number of attractive principles of conditional logic, such as the following:

$$
\begin{aligned}
& \text { Modus Ponens: }(A \Rightarrow B) \supset(A \supset B) \\
& \text { Agglomeration: }((A \Rightarrow B) \wedge(A \Rightarrow C)) \supset(A \Rightarrow(B \wedge C)) \\
& \text { Weakening the Consequent: }((A \Rightarrow B) \wedge(B \vdash C)) \supset(A \Rightarrow C) \text {. }
\end{aligned}
$$

A consequence of Lowe's account is that it also validates Hypothetical Syllogism, which Lowe takes to be intuitively valid for both indicatives and counterfactuals. So if Lowe's reduction is correct, Hypothetical Syllogism is valid for both classes of conditional. 
It is not clear how much weight such an argumentative strategy can bear, however. First, we should note that there are alternative simple logics of conditionals, including Stalnaker's (1968) and Lewis's (1986), which validate the three principles above but not Hypothetical Syllogism. So we can draw no lessons from the fact that Lowe's simple system validates these principles.

Of course, Lowe offers a reductive account of the logic of conditionals, but even if everything else were equal, this would not be a decisive consideration in favour of Hypothetical Syllogism. Moreover, everything else is not equal, since although Lowe validates the attractive principles above, he fails to validate

Reflexivity: $A \Rightarrow A$.

But surely Reflexivity is the most intuitive schema of conditional logic, and so Lowe's argumentative strategy cannot succeed, if he trades Reflexivity for Hypothetical Syllogism. ${ }^{3}$

Instead of taking Lowe's specific reduction, we could take natural language conditionals to be strict conditionals: $A \Rightarrow C=d f \square(A \supset C)$. This account, like Lowe's, offers a reduction of conditional logic which validates Modus Ponens, Agglomeration, and Weakening the Consequent, but unlike Lowe's, it validates Reflexivity as well. And this reduction still validates Hypothetical Syllogism. Unfortunately, this account, unlike Lowe's, validates three further controversial principles of conditional logic, namely

$$
\begin{aligned}
& \text { Strengthening the Antecedent: }(A \Rightarrow C) \supset((A \wedge B) \Rightarrow C) \\
& \text { Contraposition: }(A \Rightarrow C) \supset(\sim C \Rightarrow \sim A) \\
& \text { SDA: }((A \vee B) \Rightarrow C) \supset((A \Rightarrow C) \wedge(B \Rightarrow C)) .
\end{aligned}
$$

\footnotetext{
3 Lowe does, however, validate a restricted version of Reflexivity, namely $\diamond A \supset(A \Rightarrow A)$, but instances of Reflexivity are compelling even when $A$ is impossible.
} 
It would, I think, be a heroic task to argue that Hypothetical Syllogism and these further principles are valid simply on the basis that they are validated by some otherwise attractive logic of conditionals. If this strategy were plausible, then why not treat all conditionals as material conditionals? Moreover, Williamson (2007) has shown that we can subsume modal logic within the standard Stalnaker-Lewis counterfactual logic, and has further argued that it is through our knowledge of counterfactuals that we gain knowledge of metaphysical modality. Without wanting to endorse Williamson's position, it is far from clear in which direction, if any, a reduction of the logics of conditionals and modality should go. Lowe's reduction of conditional logic, then, provides no support for Hypothetical Syllogism.

\section{Morreau's Reductio of Hypothetical Syllogism}

Although Lowe's reductive strategy fails, we can make progress by examining how Hypothetical Syllogism interacts with other principles of conditional logic. In particular, Morreau (2009: 448-449) argues that if indicative conditionals validate some compelling principles of conditional logic, as well as Hypothetical Syllogism, then the indicative conditional is equivalent to the material conditional. Morreau seems to take this as a reductio of Hypothetical Syllogism for indicative conditionals

In fact, Morreau's proof generalizes to any conditional which obeys the following logical principles:

Agglomeration: $((A \Rightarrow B) \wedge(A \Rightarrow C)) \supset(A \Rightarrow(B \wedge C))$

Reflexivity: $A \Rightarrow A$ 
Weakening the Consequent: $((A \Rightarrow B) \wedge(B+C)) \supset(A \Rightarrow C)^{4}$

Conjunction Conditionalization: $(A \wedge C) \supset(A \Rightarrow C)$.

Together Reflexivity and Weakening the Consequent give the further principle

Necessary Consequent: $\mathrm{A} \Rightarrow \mathrm{T}$, for any logical truth, $\mathrm{T}$.

(From the definition of $T$, we have $A \vdash T$, and Reflexivity gives $A \Rightarrow A$, so by Weakening the Consequent, we have $A \Rightarrow T$.)

From these principles we can prove $(A \supset B) \supset(A \Rightarrow B)$ :
7. $A \supset B$
Assumption
8. $\mathrm{A} \Rightarrow \mathrm{T}$
Necessary Consequent
9. $T \Rightarrow(A \supset B)$
7, T, Conjunction Conditionalization
10. $A \Rightarrow(A \supset B)$
8, 9, Hypothetical Syllogism
11. $A \Rightarrow A$
Reflexivity
12. $A \Rightarrow(A \wedge(A \supset B))$
10, 11, Agglomeration
13. $A \Rightarrow B$
12, Weakening the Consequent
14. $(A \supset B) \supset(A \Rightarrow B)$
7, 13, Conditional Proof.

Morreau then claims that Modus Pones is uncontroversial when $(A \Rightarrow B)$ is read as an indicative conditional, and so if Hypothetical Syllogism is valid, the indicative conditional is

\footnotetext{
${ }^{4}$ As Williamson (2007: 295-296) notes, some delicacy is required in formulating Weakening the Consequent for counterfactuals when the language contains a rigidifying actually operator, @. Plausibly, $(P \equiv @ P)$ is a logical truth, but we cannot conclude from the obvious $P>P$, that $P>@ P$, since this is false when $\sim P$, contra Weakening the Consequent. Such issues are orthogonal to our concerns, however. In any case we can avoid them by replacing $P$ in the formulation Weakening the Consequent with a necessity operator, $\square$.
} 
logically equivalent to the material conditional, and so we face the paradoxes of material implication. Five points to note about Morreau's proof.

First, although many theorists endorse Modus Ponens for indicatives, some do not. McGee (1985) presents a putative counterexample to Modus Ponens for embedded indicative conditionals, and more recently, Kolodny and MacFarlane (2010) have presented a counterexample to Modus Ponens for indicative conditionals with modal consequents. Morreau could, if he wished, accept these counterexamples, and revise the conclusion of his argument accordingly. Morreau could maintain that the equivalence of a certain class of simple indicative conditionals with material conditionals represented a reductio of Hypothetical Syllogism for such conditionals. But some would resist even this result, rejecting Modus Ponens for simple indicative conditionals (e.g. Lycan 2001). Modus Ponens is, however, inessential to Morreau's reductio, as (14) alone is sufficient to generate the paradoxes of material implication. That is, (14) secures the allegedly unwelcome result that $\sim A$ or $B$ is sufficient for the truth of the indicative conditional 'if $A, B$ '.

Second, that indicative conditionals are subject to the paradoxes of material implication is a counterintuitive and surprising result, but one that others have claimed we can live with (e.g. Jackson 1987). ${ }^{5}$ So Morreau's argument against the validity of Hypothetical Syllogism for indicative conditionals may be thought not to be decisive on that account. Having said that, I do not endorse the material conditional analysis of indicatives, and nor do a number of advocates of Hypothetical Syllogism.

Third, as noted above, Morreau's proof generalizes to show that any conditional which obeys Hypothetical Syllogism and the four principles of conditional logic above is weaker than the material conditional. In particular, since the principles of conditional logic required for the proof are also plausible in the case of counterfactuals, Morreau's proof could be taken to

${ }^{5}$ See Edgington (1995) for discussion of Jackson's proposal. 
show that if counterfactuals validate Hypothetical Syllogism, then they are weaker than the material conditional. Moreover, whereas one might be prepared to accept that indicative conditionals are weaker than material conditionals, no one is prepared to accept that counterfactual conditionals are weaker than material conditionals. So if counterfactual conditionals obey the four principles above, they do not obey Hypothetical Syllogism.

Fourth, the most contentious part of the proof, for either indicative or counterfactual conditionals, is, I think, its reliance on Conjunction Conditionalization. As noted already, some reject Modus Ponens, and so will reject the stronger Conjunction Conditionalization. Moreover, even those who accept Modus Ponens need not be committed to Conjunction Conditionalization, and it has been rejected both for indicatives (e.g. Pendlebury 1989; Read 1995), and for counterfactuals (e.g. Bennett 2003; Fine 1975; McDermott 2007; and Nozick 1981). ${ }^{6}$

Morreau (2009: $448 \mathrm{n} 1$ ) notes that one might balk at Conjunction Conditionalization for indicative conditionals on the grounds that the truth of such a conditional requires a substantive connection between the antecedent and the consequent. And what is thought to go for indicatives might be thought to go for counterfactuals too. ${ }^{7}$ But, as John Pollock (1976: 35) pointed out, if a substantial connection between the antecedent and consequent is required for the truth of a conditional, then Weakening the Consequent fails. For example, if I were to press the button, the bell would ring; the bell ringing entails that the bell exists; but since there is no connection between my pressing the button and the bell's existence, 'if I were to press the button, the bell exists', is false, if a substantial connection between

\footnotetext{
${ }^{6}$ Conjunction Conditionalization is a theorem of the standard Stalnaker-Lewis logic of counterfactuals and is validated by their possible worlds semantics. A number of authors have proposed distinct possible world semantics for counterfactuals which invalidate Conjunction Conditionalization. I discuss (2009, MS) some recent proposals of this type and show them all wanting.

${ }^{7}$ Lowe (1995: 48) seems to endorse this sentiment for both indicatives and counterfactuals: "One plausible idea is that to affirm something of the form 'If $p$, then $q$ ' is to imply that there is some sort of 'necessary connection' between $p$ and $q$, in the sense that we deem it to be no accident that $p$ and the negation of $q$ are not jointly true." As I shall argue below, it is only as a result of thinking of conditionals in this way, that Hypothetical Syllogism appears plausible.
} 
antecedent and consequent is required. Pollock makes the point for counterfactuals, but it holds equally for indicatives: 'if I press the button, the bell will ring' can be true, but 'if I press the button, the bell exists' cannot be true, if a substantial connection is required (see also Stalnaker 1968). Weakening the Consequent is not in dispute, however, and as I shall argue in the next section, a substantial connection between antecedent and consequent is but one way in which a conditional can be true, so Conjunction Conditionalization is not to be rejected on these grounds.

Nevertheless, Conjunction Conditionalization is the most controversial element of the proof, and so the case against Hypothetical Syllogism would be much improved, if it were not required. Moreover, advocates of Hypothetical Syllogism, such as Lowe (1995), von Fintel (2001), and Gillies (2007), accept the other principles required to make trouble for Hypothetical Syllogism, but reject Conjunction Conditionalization. ${ }^{8}$ So as it stands, advocates of Hypothetical Syllogism will take Morreau's proof, not as a reductio of Hypothetical Syllogism, but instead as a reductio of Conjunction Conditionalization.

Finally, Morreau's proof does not require Conjunction Conditionalization in its full generality, but only something like the following restricted version

Conjunction Conditionalization*: $(\square A \wedge C) \supset(A \Rightarrow C)$.

Recognising this does not help, however. First, any reasons for rejecting Conjunction Conditionalization are likely to extend to rejecting Conjunction Conditionalization*, and so those who reject Conjunction Conditionalization will reject Conjunction Conditionalization* as well. For example, many authors reject Conjunction Conditionalization on the basis of

\footnotetext{
${ }^{8}$ As noted above, Lowe (1995) limits Reflexivity to conditionals with possible antecedents, but the paradoxes of material implication are just as worrying when the antecedent of a conditional is possible. The question, then, is whether Lowe would concede that the antecedent is possible in Morreau's proof. We return to this question below.
} 
putative counterexamples, such as McDermott's (2007). Consider a situation in which a coin is to be tossed twice; before it is tossed I bet that it will come up heads both times; it does and I win. Conjunction Conditionalization licenses the claim that if at least one head had come up, I would have won. McDermott claims, however, that this is false and so Conjunction Conditionalization is to be rejected. But the conditional 'if none or more heads had come up, I would have won' scarcely sounds any better. But if this too is false, then given its necessary antecedent, Conjunction Conditionalization* is invalid.

Second, if there are some true $A$ and $C$ such that both $\sim(A \Rightarrow C)$ and $\sim(\sim A \Rightarrow C)$, as surely there are for those who deny Conjunction Conditionalization, then the following principle fails, if Conjunction Conditionalization* is valid but Conjunction Conditionalization is not:

$$
\mathrm{SDA}^{*}:((A \vee B) \Rightarrow C) \supset((A \Rightarrow C) \vee(B \Rightarrow C)) \text {. }
$$

This is because, by Conjunction Conditionalization*, $(A \vee \sim A) \Rightarrow C$ is true when $C$ is true, but in our scenario, neither $A \Rightarrow C$ nor $\sim A \Rightarrow C$ are true.

But SDA* is seemingly obvious for both indicative and counterfactual conditionals, and is, in any case, accepted by those who embrace Hypothetical Syllogism. So rejecting Conjunction Conditionalization whilst accepting Conjunction Conditionalization* is not an option.

As it stands, then, Morreau's reductio of Hypothetical Syllogism will be rejected by its defenders, since it relies on the controversial Conjunction Conditionalization (or its cousin Conjunction Conditionalization*). Nevertheless, Morreau's proof does offer us a way forward in resolving the dispute over Hypothetical Syllogism. The only role that Conjunction Conditionalization plays in the proof is in establishing (9) $T \Rightarrow(A \supset B)$. So if we have some other way of deriving (9), the proof need not appeal to the contentious Conjunction 
Conditionalization. And we can, I shall argue, establish a restricted version of (9), at least for counterfactuals, without relying on Conjunction Conditionalization. Further, once we see how to do this, this insight alone shows that Hypothetical Syllogism is invalid.

\section{Irrelevant Conditionals}

When first theorising about counterfactuals, it is easy to think that some connection between the antecedent and the consequent of the conditional is required for it to be true. And it is true that many counterfactuals are like that, including the premises of the putative counterexamples to Hypothetical Syllogism above. But this is only one ground for the truth of a counterfactual. Counterfactuals are also true when the consequent is true and the antecedent is irrelevant to whether or not the consequent obtains. For example, we might say of a witch doctor

'It would not rain if he did not do a rain dance, but it would not rain if he did either.' This conjunction of two simple subjunctives expresses the lack of a connection rather than the presence of one. (Pollock 1976: 26; cf. McDermott 2007: 334).

To endorse a pair of conditionals of the form 'if $A$ had been the case, $C$ would have been the case' $(A>C)$, and 'if $A$ had not been the case, $C$ would have been the case' $(\sim A>C)$ is one way of saying that $C$ is true and there is no connection between whether or not $A$ obtains and whether or not $\mathrm{C}$ obtains (that is, $\mathrm{A}$ is irrelevant to whether or not $\mathrm{C}$ obtains). More generally, we can say that when $A$ is irrelevant to whether or not $C$ obtains, then either $((A)$ $C) \wedge(\sim A>C))$ or $((A>\sim C) \wedge(\sim A>\sim C))$, depending on whether or not $C$ is true or false. On the basis of these facts, Pollock rightly concludes that 
subjunctives do not express a relation of necessitation between their antecedent and consequent. Rather, the presence of such a connection is just one ground for asserting a simple subjunctive. It seems that there are basically two ways that a simple subjunctive can be true. On the one hand, there can be a connection between the antecedent and consequent so that the truth of the antecedent would bring it about ... that the consequent would be true. On the other hand, a simple subjunctive can be true because the consequent is already true and there is no connection between the antecedent and consequent such that the antecedent's being true would interfere with the consequent's being true (Pollock 1976: 26).

Let us call conditionals that are true in virtue of some connection between the antecedent and consequent 'realization conditionals' since the antecedent in some way realizes the consequent, and let us call conditionals with antecedents that are irrelevant to the obtaining of its already true consequent 'irrelevant conditionals'. Pollock's claim then, is that both realization conditionals and irrelevant conditionals figure amongst the true counterfactuals.

Irrelevant conditionals are just as much a part of our practice of using counterfactuals as are realization conditionals, as the quote form Pollock above shows. Moreover, it is in virtue of such irrelevant conditionals that we endorse Morgenbesser's conditional 'if you had bet on heads, you would have won' said to someone who was offered a bet they declined when the coin subsequently landed heads. ${ }^{9}$ That is, 'if you had bet on heads, the coin would have landed heads' is a true irrelevant conditional. But from this it follows from the instance of Reflexivity 'if you had bet on heads, you would have bet on heads', and Agglomeration that 'if you had bet on heads, you would have bet on heads and the coin would have landed heads' which is tantamount to the Morgenbesser conditional.

\footnotetext{
${ }^{9}$ We should note, however, that despite the widespread acceptance of our semantic claim that irrelevant conditionals are true, Phillips (2007) rejects it in indeterministic contexts. As we shall see below though, the arguments of this paper go through even if we assume determinism.
} 
I argue in the sequel that by appealing to the truth of irrelevant conditionals and counterfactuals which turn on them, like the Morgenbesser conditional above, we can show, without appealing to Conjunction Conditionalization, that Hypothetical Syllogism is invalid for counterfactuals.

\section{Morreau's Proof without Conjunction Conditionalization}

Consider a case in which i) $\mathrm{C}$ is irrelevant to whether or not $\mathrm{A}$ obtains, ii) $\mathrm{C}$ is irrelevant to whether or not B obtains, and iii) $A \supset B$. In such a situation, the following irrelevant conditionals are then true

15. $C>(A \supset B)$

16. $\sim C>(A \supset B)$.

Proof: Assume $\sim$ A. Given assumption (i), we have $C>\sim$ A. By Weakening the Consequent we then have, $C>(A \supset B)$. Now assume $B$. By analogous reasoning from assumption (ii) we have $C>B$, and then, by Weakening the Consequent, we have $C>(A \supset B)$. So given assumption (iii), we have, by reasoning by cases, $C>(A \supset B)$. Similar reasoning yields $\sim C>$ $(A \supset B)$.

(15) and (16) together yield

17. $(C \vee \sim C)>(A \supset B)$

via the following theorem of counterfactual logic which advocates of Hypothetical Syllogism accept (see for instance Lowe 2010: 287): 
Antecedent Disjunction Introduction: $((A>B) \wedge(C>B)) \supset\left((A \vee C)>B .{ }^{10}\right.$

So by using irrelevant conditionals and Antecedent Disjunction Introduction, we can establish (17) for any A, B, and C which satisfy our assumptions (i)-(iii).

Now, as $C \vee \sim C$ is a logical truth, we can replace (8) and (9) in Morreau's proof with $A>(C \vee$ $\sim$ C) (which follows from Necessary Consequent) and (17) respectively. This, then, is a version of Morreau's proof of (14) for counterfactuals which does not employ Conjunction Conditionalization, but instead relies upon Antecedent Disjunction Introduction, and the truth of irrelevant conditionals. And as it relies only on uncontroversial logical principles and the claim that irrelevant conditionals are true, we can take this revised proof as a reductio of Hypothetical Syllogism for counterfactuals.

Of course, this proof only establishes that the counterfactual conditional is weaker than the material conditional, on the assumption that for every true instance of $A \supset B$, there is some $C$, such that (i) and (ii) hold. That is, on the assumption that for every true instance of $A \supset B$, there is some $\mathrm{C}$ which is irrelevant to whether or not $\mathrm{A}$ obtains and irrelevant to whether or not B obtains. If this assumption holds, we can establish all instances of (17), and thus, by the revised version of Morreau's proof, $(A \supset B) \supset(A>B)$. So the question is, does this assumption hold?

Reconsider Morgenbesser's conditional 'if you had bet on heads, you would have won'. As noted above, the thought behind this conditional is that the result of the coin toss is independent of your betting behaviour, so that whether or not you bet on heads is irrelevant to whether or not the coin lands heads. A consequence of this is that, given that the coin did

\footnotetext{
10 In fact we need only appeal to the version of Antecedent Disjunction Introduction that holds when C is the negation of $A$.
} 
lands, it would still have landed heads, if you had bet on heads, and also that it would have landed heads, if you had not bet on heads. But there is nothing special about coin tosses: our betting behaviour is, in general, irrelevant to whether some other event happens or not. So, if we can assume that for every instance of $A \supset B$, someone's betting behaviour is irrelevant to whether or not A obtains and whether or not B obtains, we can establish an instance of (17) for every true instance of $A \supset B$. We can then complete the reductio of Hypothetical Syllogism using the revised version of Morreau's proof outlined above.

Unfortunately, our assumption about betting behaviour does not hold in full generality. For instance, let $\sim A$ be a proposition that holds only at the world of evaluation, so that if $C$ is true, then $\sim C>\sim A$ is false. In such a case, our route to (16), and hence (17), is blocked. Although there may be some other route to this instance of (17), let us assume that we cannot establish every instance of (17). As a result, what the argument establishes, assuming Hypothetical Syllogism, is not that counterfactuals are weaker than material conditionals, but merely that there are many particular truths of the form $(A \supset B) \supset(A>B)$. That is, in many cases of $A \supset B$ it is safe to assume that someone's betting behaviour is irrelevant to whether or not A obtains and irrelevant to whether or not B obtains and so in these cases we can establish $(A \supset B) \supset(A>B)$. But this still constitutes a reductio of Hypothetical Syllogism for counterfactuals, since $A \supset B$ is not in general sufficient for the truth of $A>B$. These cases, then, show that Hypothetical Syllogism is invalid for counterfactuals. Moreover, in order to show the invalidity of Hypothetical Syllogism, there need only be one false instance of ( $A$ $B) \supset(A>B)$ forthcoming from the argument above.

\section{Another Reductio of Hypothetical Syllogism}


Morreau's reductio, then, can be buttressed to avoid relying on Conjunction

Conditionalization. ${ }^{11}$ In fact, once we have irrelevant conditionals on the scene, we can offer a simpler proof of the invalidity of Hypothetical Syllogism for counterfactuals. Assuming (i) that $C$ is irrelevant to whether or not $A$ obtains, and (ii) $\sim A$, we have the following:

18. $C>\sim A$

19. $\sim C>\sim A$.

By Antecedent Disjunction Introduction, (18) and (19) license

20. $(C \vee \sim C)>\sim A$.

But given that

21. $A>(C \vee \sim C)$

follows from Necessary Consequent, Hypothetical Syllogism allows us to derive

22. $A>\sim A$

from (20) and (21). All parties to the debate agree, however, that (22) cannot be true unless $\sim A$ is necessary. But apart from there being some C, such that (20) and (21) hold, A has

11 In fact one can employ the considerations of this paper to argue for the truth of Conjunction Conditionalization (see Walters 2009; Walters and Williams Forthcoming) and so support Morreau's original proof. Those arguments rely on the following theorem of the standard logic of counterfactuals: $((A>B) \wedge(B>A) \wedge(A>C)) \supset(B>C)$. This theorem has been disputed (see Walters 2011 for some discussion), but in the present context it cannot be denied as it is weaker than Hypothetical Syllogism. The argument for Conjunction Conditionalization also requires that irrelevant conditionals are true under indeterminism, and that for every true $A$ and $B$, there is some $C$ irrelevant to the obtaining of $A$ and irrelevant to the obtaining of B. The present argument for the invalidity of Hypothetical Syllogism, however, does not require the truth of irrelevant conditionals under indeterminism, and requires only enough irrelevancy to produce one false instance of $(A \supset B) \supset(A>B)$. 
been selected arbitrarily and so can be contingent. So we have here a reductio of Hypothetical Syllogism, employing our semantic claim about irrelevant conditionals, Antecedent Disjunction Introduction, and Necessary Consequent.

\section{An Objection to these Proofs}

We argued above that Hypothetical Syllogism licenses false instances of (14) and (22), and so is to be rejected. If there are to be false instances of (14), then there must be instances where $B$ is not necessary, since when $B$ is necessary $A>B$ follows by Necessary Consequent. But it is consistent with our assumption of $A \supset B$ in the revised proof of (14), that $B$ is false or otherwise not necessarily true, and so the existence of false instances of (14) is not threatened.

Similarly, if A is impossible, our proof of (14) does not threaten Hypothetical Syllogism. As we noted above, Lowe rejects Reflexivity for impossible propositions, and so he will reject (11). As a result, neither Morreau's original nor the revised proof of (14) goes through, unless A is possible. On the other hand, those who endorse Reflexivity will allow that (14) is true when A is impossible, if they accept that counterfactuals with impossible antecedents are automatically true. In such circumstances we cannot retreat to (22), since (22) is true when $A$ is impossible in virtue of its necessary consequent. But there need be no sense in which $A$ is impossible, other than that it did not in fact obtain. $A$, we may stipulate, is consistent with the laws of nature and highly probable. Of course, if the scenario under consideration is deterministic, then given that $A$ does not obtain, $A$ is inconsistent with the laws of nature plus the initial state of the universe. But we do not think that simply in virtue of determinism $A>\sim A$ is true whenever $\sim A$. In any case, we can stipulate that (18) to (22) are to be considered against a back-drop of indeterminism. In this case, A need not even be 
inconsistent with the conjunction of the laws of nature and the initial state of the universe. So it is simply not plausible to claim that $A$ is impossible. ${ }^{12}$

In response to Morreau's putative counterexample to Hypothetical Syllogism, Lowe (2010: 288) contends that claims of the form of (17) and (20) are saying "that something wouldn't have happened come what may". He then rightly notes that to assert "that something wouldn't have happened come what may is very plausibly tantamount to asserting that, in the relevant sense of 'could', it just couldn't have happened". If Lowe is correct, (22) does not represent a counterexample to Hypothetical Syllogism, since, for Lowe (20) says that A wouldn't have happened come what may, and so $\sim A$ is necessary. But the necessity of $\sim A$ means that (22) is true. Similarly, if (17) says that $A \supset B$ is necessary, then the advocate of Hypothetical Syllogism could accept our revised proof of (14), if they accept that $\square(A \supset B)$ entails $A>B$.

This line of response is unconvincing, however, as Lowe is incorrect about conditionals like (17) and (20). Although (20) is equivalent to the claim that A would not happen come what may on Lowe's own semantics, this merely highlights the implausibility of his semantics. There is nothing in the structure of the example to prevent $A$ from being consistent with the laws of nature and indeed with the laws of nature and the state of the universe shortly before A was the case. Indeed, A may have been highly probable at this time.

To see the implausibility of Lowe's claim, compare it with Mill's conditional characterization of necessity which rests on a much more intuitive notion of 'come what may':

If there be any meaning which confessedly belongs to the term necessity, it is unconditionalness. That which is necessary, that which must be, means

\footnotetext{
12 Of course, if we appeal to indeterminism, then we need the claim, which Phillips (2007) rejects, that irrelevant conditionals are true in indeterministic contexts.
} 
that which will be, whatever supposition we may make in regard to all other things. (Mill 1882: 245 my underlining)

Transferring Mill's thought from indicative to counterfactual conditionals, we have

23. $\square \mathrm{C} \equiv \forall \mathrm{P}(\mathrm{P}>\mathrm{C})^{13}$

But intuitively (20) can be true without the right-hand side of (23) being true for, typically, there will be some $B$, such that ' $B>A$ ' is false, even when $(C \vee \sim C)>A$. That is, we cannot conclude that all facts are irrelevant to whether or not $A$ obtains from the fact that $C$ is irrelevant to whether or not A obtains. So it is simply not plausible for Lowe to claim that $\sim A$ is in any sense necessary, other than that it actually obtained. And whatever we want to say about indicatives, the falsity of $A$ is not sufficient for the truth of $A>\sim A$.

\section{Improving the Proof}

The two proofs of the invalidity of Hypothetical Syllogism above relied on further principles of counterfactual logic, which although extremely intuitive, offer the defender of Hypothetical Syllogism room for manoeuvre. But if we consider a case where we have two-way independence, that is, where $\mathrm{C}$ is irrelevant to whether or not $\mathrm{A}$ obtains, and $\mathrm{A}$ is irrelevant to whether or not $\mathrm{C}$ obtains, then we have a proof of the invalidity of Hypothetical Syllogism in propositional logic alone. Consider the following set-up: neither $A$ nor $C$ is the case and $A$ is irrelevant to whether or not $C$ obtains, and $\sim C$ is irrelevant to whether or not $A$ obtains. In these circumstances the following are true irrelevant conditionals

24. $\sim C>\sim A$

25. $A>\sim C$.

${ }^{13}$ Cf. Lewis (1986: 23) and Williamson (2007: 159). 
From (24) and (25) by Hypothetical Syllogism, we can conclude.

26. $A>\sim A$.

But as above, $\sim \mathrm{A}$ can be contingent, and so it is not plausible to claim that (26) is true. So Hypothetical Syllogism is to be rejected.

Lowe might respond that in order to know that (25) is true, we would need to know that $\sim \mathrm{C}$ is true. But in such a case, we would have no use for (24) since we wouldn't use a counterfactual when we know that its antecedent is true. ${ }^{14}$ Of course, none of this affects the truth or falsity of (24).

In any case, we could consider a context in which we only know that $\sim A$ and so we do have a use for (24). Of course, we can only be confident of (25) if we know $\sim C$, so to properly assent to (25) we need to know $\sim \mathrm{C}$. But all this is consistent with learning that $\sim \mathrm{C}$ once we have already assented to (24). So unless learning that $\sim C$ changes the context in some relevant way, then it seems we have here a counterexample to Hypothetical Syllogism. Moreover, it seems that, at least as von Fintel, Gillies, and Lowe construe things, learning that $\sim \mathrm{C}$ does not change the context in any relevant way.

Of course, (24) has a true antecedent and we may concede that such counterfactuals are, in some sense, defective. ${ }^{15}$ But what we are interested in here is truth, not unassertability or some other measure of defectiveness, and so (24) and (25), show the invalidity of Hypothetical Syllogism.

${ }^{14}$ This claim is not true in its full generality, but we'll grant it here (see Edgington 1995: 240 for some discussion).

${ }^{15}$ Lowe cannot object to my employment of (24) since he himself employs an analogous conditional in his rejection of Morreau's putative counterexample to Hypothetical Syllogism (Lowe 2010: 287-288) see $\S 6$ above. 
In any case, we can avoid these issues of assertability and knowledge of the truth of the antecedent. When we have two-way independence between $A$ and $C$, then we have the following four claims, even when we do not know whether or not $\mathrm{A}$ or $\mathrm{C}$ obtain:

27. $C \supset(A>C)$

28. $\sim \mathrm{C} \supset(\sim \mathrm{A}>\sim \mathrm{C})$

29. $A \supset(\sim C>A)$

30. $\sim A \supset(C>\sim A)$.

It is trivial to show that the claims below follow from (27)-(30), if Hypothetical Syllogism is valid:

31. $(A \& C) \supset(\sim C>C)$ by 27,29

32. $(A \& \sim C) \supset(\sim A>A)$ by 28,29

33. $(\sim A \& C) \supset(A>\sim A)$ by 27,30

34. $(\sim A \& \sim C) \supset(C>\sim C)$ by $28,30$.

But given that

35. $(A \& C) \vee(A \& \sim C) \vee(\sim A \& C) \vee(\sim A \& \sim C)$

is a logical truth, we know that something of the form $\phi>\sim \phi$ follows from (31) to (34). And again $\sim \phi$ here can be a contingent truth. So if we accept that irrelevant conditionals are true, at least in a context in which no counterfactual has been asserted, then we know that Hypothetical Syllogism is invalid for counterfactuals. 


\section{Context-Sensitivity}

We argued in $\S 4-\S 7$ that we can demonstrate that Hypothetical Syllogism is invalid, by appealing to a semantic claim, namely that irrelevant conditionals are true. One might be worried, however, about what such proofs could establish. Above I have provided abstract arguments in schematic terms. But given the context-sensitivity of natural language, and that advocates of Hypothetical Syllogism appeal to such context-sensitivity in order to explain away putative counterexamples, we may not be satisfied that Hypothetical Syllogism is invalid until presented with a concrete example that cannot be explained away by appeals to context.

Before I provide such a counterexample, I want to note a general point for those appealing to context-sensitivity in defending Hypothetical Syllogism. Contextual defences of Hypothetical Syllogism usually appeal to features of the context in which the counterfactuals in question are asserted. But the final proof of the last section showed that Hypothetical Syllogism is invalid even in the absence of the assertion of any counterfactuals. Rather the counterfactuals were embedded within material conditionals. The contextualist, then, cannot appeal to features of the context of assertion to explain away the proof. As such the precise accounts of Lowe (1990), von Fintel (2001), and Gillies (2007) must be abandoned.

Let me now provide counterexamples to Hypothetical Syllogism employing irrelevant conditionals, in which there is no tension in asserting both premises and rejecting the conclusion within the same context. ${ }^{16}$ Consider the following

36. If I had lived on the moon, I would have drank freeze-dried tea

37. But if I had drank freeze-dried tea, I would not have lived on the moon

${ }^{16}$ The clearest examples employ one irrelevant conditional and one realization conditional, so I focus on these. 
(37) is a true irrelevant conditional, and (36) could be true under certain circumstances. But if Hypothetical Syllogism is valid we can conclude the false

38. If I had lived on the moon, I would not have lived on the moon.

Of course, living on the moon is a far-fetched possibility, but it is not impossible. A more homely example: from

39. If I had been an inch taller than I actually am, I would have seen the show

and the irrelevant conditional

40. If I had seen the show, I would not have been an inch taller than I actually am

we cannot conclude

41. If I had been an inch taller than I actually am, I would not have been an inch taller than I actually am. ${ }^{17}$

In these examples, it is clear, I think, that there are contexts in which both of the premises are assertable, so Lowe's response fails. Moreover, we are not bound to wonder whether we are claiming that, for example, my being an inch taller is impossible, and so Wright's response fails. These examples are perfectly felicitous and show none of the strains of earlier examples which employed a pair of realization conditionals. There is, then, no work for the contextualist to do. A contextualist response to these counterexamples is seriously undermotivated and would represent an ad hoc attempt to save Hypothetical Syllogism. In

${ }^{17}$ Adapted from Moss (2012). 
the face of these cases, and the proofs above, we should simply accept the invalidity of Hypothetical Syllogism for counterfactuals, something that is not in conflict with any of our pre-theoretical judgments on the matter.

\section{Related Argument Forms}

As well as Hypothetical Syllogism, Stalnaker and Lewis claim that the following two related argument forms are also invalid for counterfactuals

$$
\begin{aligned}
& \text { Antecedent Strengthening: }(A \Rightarrow C) \supset((A \& B) \Rightarrow C) \\
& \text { Contraposition: }(A \Rightarrow C) \supset(\sim C \Rightarrow \sim A) .
\end{aligned}
$$

Lowe himself rejects both Antecedent Strengthening and Contraposition in their full generality, since he requires either the possibility of the antecedent or the necessity of the consequent of a counterfactual for it to be true. Nevertheless, Lowe accepts suitably amended versions of Antecedent Strengthening and Contraposition, and advocates of simple strict conditional accounts of counterfactuals, such as von Fintel and Gillies, accept Antecedent Strengthening and Contraposition in their full generality.

Now just as Morreau showed that Hypothetical Syllogism is incompatible with Conjunction Conditionalization, we can show that Antecedent Strengthening and Contraposition are also invalid for a conditional, $\Rightarrow$, which obeys Conjunction Conditionalization. Assume that $C$ is true, then we have
42. $\mathrm{T} \Rightarrow \mathrm{C}$
Conjunction Conditionalization
43. $(T \wedge A) \Rightarrow C$
(42), Antecedent Strengthening
44. $A \Rightarrow C$
(43), Substitution of Logical Equivalents 
But the truth of the consequent is not in general sufficient for the truth of a counterfactual, and so Conjunction Conditionalization and Antecedent Strengthening cannot jointly be maintained.

Similarly, from (42) by Contraposition we have

$$
\text { 45. } \sim \mathrm{C} \Rightarrow \sim \mathrm{T}
$$

which can only be true if $C$ is necessary. But again nothing prevents $C$ from being a contingent truth.

Of course, the defender of Antecedent Strengthening and Contraposition rejects Conjunction Conditionalization, but we can reinstate these proofs, by using the considerations above to establish $(A \vee \sim A)>C$ and then using this in place of (42) and Conjunction Conditionalization in the proofs above.

The invalidity of Antecedent Strengthening cannot be avoided by denying Substitution of Logical Equivalents. From $A>C$, Antecedent Strengthening gives $(A \wedge \sim C)>C$ which is false when $\sim C$ is compossible with $A$ (cf. Stalnaker 1987: 124). Now when A is irrelevant to whether or not $\mathrm{C}$ obtains, and $\mathrm{C}$ is irrelevant to whether or not $\mathrm{A}$ obtains, we have a particularly vivid instance of this failure. In this case we have both $A>C$ and $\sim C>A$. But given that (i) $\sim C>A$ requires that $\sim \mathrm{C}$ and $\mathrm{A}$ be compossible, unless $\sim \mathrm{C}$ is impossible and (ii) nothing in the example requires that $(\sim C \wedge A)$ be impossible, then there will be instances where $A$ and $\sim C$ are compossible, and so instances where $(A \wedge \sim C)>C$ is false. ${ }^{18}$ Such

\footnotetext{
${ }^{18}$ This argument relies on the following truth about counterfactuals: $(A>C) \supset(\diamond A \supset \diamond C)$. Given
} Reflexivity and Agglomeration, this rules out the truth of $(A \wedge \sim C)>C$ when $A$ and $\sim C$ are 
instances are sufficient to invalidate Antecedent Strengthening. So here we see that even without Conjunction Conditionalization Hypothetical Syllogism, Antecedent Strengthening and Contraposition are invalid for counterfactuals. ${ }^{19}$

\section{Indicative Conditionals}

Morreau initially presented his proof as a reductio of Hypothetical Syllogism for indicative conditionals. As I noted above, there were two problems with Morreau's proof. First, many are happy to accept the reductio and adopt a material conditional treatment of indicative conditionals. Second, those who do not embrace the reductio may well balk at Conjunction Conditionalization.

None of the proofs above rely on Conjunction Conditionalization, and only the first relied on our target conditional not being weaker than the material conditional. So this opens up the possibility that we can adapt the above proofs to show the invalidity of Hypothetical Syllogism for indicative conditionals too.

Above, following Pollock, I defended the truth of irrelevant counterfactual conditionals, but I think the analogous claim is plausible in the case of both forward-looking and backwardlooking indicatives as well: if you go the party, I shan't go. Don't get me wrong though, I shan't go to the party, if you don't go either. If you came in late last night, I didn't hear you, and if you didn't come in late last night, I didn't hear you. In any case, as with the argument above concerning counterfactuals, the semantic claim concerning these types of conditionals is not needed in its full generality. To show the invalidity of Hypothetical Syllogism we need but an instance of the argument forms above.

compossible. Again given Reflexivity and Agglomeration, it also entails that $A$ and $\sim C$ are compossible given both $\sim \mathrm{C}>\mathrm{A}$ and $\diamond \sim \mathrm{C}$.

19 The proof of the invalidity of Contraposition cannot be avoided by denying Antecedent Disjunction Introduction in the proof of $(A \vee \sim A)>C$, since given Contraposition, Antecedent Disjunction Introduction is equivalent to Agglomeration which cannot be denied. 
One problem with extending the arguments above to the case of indicatives is that one might be happy to accept 'if $\sim A, A$ ' as a way of saying that the antecedent is false. This has much more plausibility than accepting $\sim A>A$ in the cases above and insisting that this shows $A$ is impossible. One can simply treat instances of 'if $\sim A, A$ ' as special cases of monkey's uncle conditionals, such as 'if you're the next Kant, then I'm a monkey's uncle'.

Nevertheless, we can show that advocates of Hypothetical Syllogism are committed to many instances of $A \supset B$ being sufficient for the indicative conditional 'if $A, B$ ', and we can do that without relying on Conjunction Conditionalization. Indeed, we can show that the truth of the consequent is sufficient for the truth of any conditional which obeys Hypothetical Syllogism. Consider the following proof where B obtains independently of C:

46. $B$

47. $C \Rightarrow B$

48. $\sim \mathrm{C} \Rightarrow \mathrm{B}$

49. $(C \vee \sim C) \Rightarrow B$

50. $A \Rightarrow(C \vee \sim C)$

51. $A \Rightarrow B$

52. $B \supset(A \Rightarrow B)$
Assumption

(46) Irrelevancy of C

(46) Irrelevancy of $\sim C$

(47), (48), Antecedent Disjunction Introduction

Necessary Consequent

(49), (50), Hypothetical Syllogism

(46), (51), Conditional Proof

The truth of the consequent is obviously not sufficient for the truth of a counterfactual, and is seemingly just as obviously not sufficient for the truth of an indicative either. Of course, some people will embrace this conclusion in the case of indicatives, but for those who don't, Hypothetical Syllogism is untenable, regardless of whether or not they accept Conjunction Conditionalization. 


\section{Conclusion}

It is overwhelmingly plausible that irrelevant conditionals are true (at least in deterministic contexts). If we accept this fact, then we can show that Hypothetical Syllogism and related argument forms are invalid for counterfactuals. This insight also allows us to provide newstyle counterexamples to Hypothetical Syllogism for counterfactuals, which are impervious to traditional contextualist strategies to save Hypothetical Syllogism. And these same considerations show that advocates of Hypothetical Syllogism for indicatives are committed to the paradoxes of material implication.

Lowe (2010: 289) contends that the dispute over Hypothetical Syllogism "will never be conclusively settled merely by piecemeal appeal to alleged 'counter-examples', but only, if at all, on the basis of broader, systemic considerations". This, I suggest, is because Lowe has limited himself to a restricted diet of examples. I hope to have shown that if we instead adopt a more balanced diet, comprised of a fuller range of cases, we will find more straightforward counterexamples to Hypothetical Syllogism. ${ }^{20}$

\section{References}

Bennett, J. 2003: A Philosophical Guide to Conditionals. Oxford: OUP.

Edgington, D. 1995: On Conditionals. Mind 104: 235-329.

Fine, K. 1975: Critical Notice of David Lewis's Counterfactuals. Mind 84: 451-458. Reprinted in his Modality and Tense (2005) Oxford: OUP. pp. 357-365.

20 Thanks to Nick Jones, Ian Phillips, and especially an anonymous referee for comments. 
von Fintel, K. 2001: Counterfactuals in a Dynamic Context. In Michael Kenstowicz (ed.), Ken Hale: A Life in Language, pp. 123-52. Cambridge, MA: MIT Press.

Gillies, A. 2007: Counterfactual Scorekeeping. Linguistics and Philosophy 30: 329-60.

Jackson, F. 1987: Conditionals. Oxford: Basil Blackwell.

Kolodny, N. and MacFarlane, J. 2010: Ifs and Oughts. Journal of Philosophy 107: 115-143.

Lewis, D. 1986: Counterfactuals, revised edn. Oxford: Basil Blackwell.

Lowe E. J. 1983: A Simplification of the Logic of Conditionals. Notre Dame Journal of Formal Logic 24: 357-366.

Lowe, E.J. 1990: Conditionals, Context and Sensitivity. Analysis 50: 80-87.

Lowe, E.J. 1995: The Truth About Counterfactuals. The Philosophical Quarterly 45: 41-59.

Lowe, E.J. 2010: Another Dubious Counterexample to Conditional Transitivity. Analysis 70: 286-289.

Lycan, W. 2001: Real Conditionals. Oxford: OUP.

McDermott, M. 2007: True Antecedents. Acta Analytica 22: 333-335.

McGee, V. 1985: A Counterexample to Modus Ponens. Journal of Philosophy 82: 462-471. 
Mill, J. S. 1882: A System Of Logic, Ratiocinative And Inductive. New York: Harper \& Brothers, Publishers. Available at http://www.gutenberg.org/files/27942/27942-h/27942$\underline{\text { h.html }}$

Morreau, M. 2009: The Hypothetical Syllogism. Journal of Philosophical Logic 38: 447-464.

Moss, S. 2012: On the Pragmatics of Counterfactuals. Nous 46: 561-586.

Nozick, R. 1981: Philosophical Explanations. Oxford: OUP.

Pendlebury, M. 1989: The Projection Strategy and the Truth Conditions of Conditional Statements. Mind 98: 179-205.

Phillips, I. 2007: Morgenbesser cases and closet determinism. Analysis 67: 42-49.

Pollock, J. 1976: Subjunctive Reasoning. Boston, MA: Reidel.

Read, S. 1995: Conditionals and the Ramsey test. Proceedings of the Aristotelian Society, 69: 47-65.

Stalnaker, R. 1968: A Theory of Conditionals. In Studies in Logical Theory: American Philosophical Quarterly Monograph Series, no. 2, ed. Nicholas Rescher, pp. 98-112. Oxford: Blackwell.

Stalnaker, R. 1987: Inquiry. Cambridge Ma: MIT Press.

Walters, L. 2009: Morgenbesser's Coin and Counterfactuals with True Components. Proceedings of the Aristotelian Society CIX: 365-379. 
Walters, L. 2011: Reply to Ahmed. Proceedings of the Aristotelian Society CXI: 123-133.

Walters, L. MS: Possible Worlds Semantics and True-True Counterfactuals.

Walters, L. and Williams, J.R.G. 2013: An Argument for Conjunction Conditionalization. The Review of Symbolic Logic 6: 573-588.

Williamson, T. 2007: The Philosophy of Philosophy. Oxford: Blackwell.

Wright, C. 1983: Keeping track of Nozick. Analysis 43: 134-40.

Wright, C. 1984: Comment on Lowe. Analysis 44: 183-185. 